\title{
Electric Vehicle Charging Load Forecasting Based on ACO and Monte Carlo Algorithms
}

\author{
Tianyi Qu ${ }^{1, a}$ and Xiaofang Cao ${ }^{1, b}$ \\ ${ }^{1}$ School of Management, Xuzhou institute of Technology, Xuzhou, Jiangsu, China 221008 \\ ajdbh2001@163.com, bcxfxzit@163.com
}

Keyword: Electric vehicle; Charging load; Ant colony algorithm; Monte carlo simulation algorithm; Fault line selection; Ground fault

\begin{abstract}
In this paper, according to "Electric Vehicle Charging Infrastructure Development Guide" (2015-2020) planning and "The Plan of Distribution Network Construction and Transformation" (2015-2020), intelligent ant colony algorithm and Monte Carlo algorithm is used to predict respectively the total electricity loads of electric vehicles and characteristic of whether the loads are in line with the curve in a region. Predicted results have certain reference value for the study of the future growth of China's electric vehicle charging load.
\end{abstract}

\section{Introduction}

With the continuous development of China's economic society, car ownership keeps rising. Developing electric vehicles can accelerate alternative fuels and reduce vehicle emissions. What's more, it is of great significance to ensure energy security, promote energy saving, control air pollution and realize the change from a big country to a powerful country in automobile industry.

Charging infrastructure includes all types of centralized power station and decentralized chargingpiles. Perfect charging infrastructure system is an important guarantee of the popularity of electric vehicles. Vigorously promoting the further charging infrastructure is an urgent task to accelerthe application electric vehicles, which is also a significant strategic initiatives to promote the consumption of energy revolution.

At present, the charging infrastructure is still in the initial stage at home and abroad, and electric vehicles and charging technology still exist big uncertainty. Electric car industry is still in early phase of development, and the key technology of power battery and charging is advancing rapidly, there are different technical solutions corresponding to large differences of the charging requirements, which increase the difficulties of the construction of charging infrastructure and its management, meanwhile increasing a lot of difficulties for the load forecast of the grid side, distribution network planning, power quality, system stability, system optimization and other aspects, putting forward new requirements for distribution network.

The large-scale charging facilities that connected to the grid belongs to the high-power impact load, thus resulting in the redistribution of power load, causing the variation of power flow and increase of grid losses. Meanwhile, as the charging facilities are non-linear loads, and there exists various equipments, standard systems also need to be improved. Thus, with the continuous expansion of the ownership of electric vehicles and national related planning, a large number of charging facilities continue to access to meet the charging requirements of a large number of electric vehicles. However, that large-scale charging facilities connect to grid without planning is bound to cause adverse effects on the distribution network loads, voltage, grid loss, three-phase imbalance, harmonic components, sags, flicker and other aspects. Visibly, if those affects and the problem of standard specification system of charging infrastructure are not perfectly solved, which are inevitably affect the coordinated development of charging infrastructure and electric vehicles.

Given that, that conducting the research of forecasting of the large-scale charging loads not only is of great significance to ensure security, reliability, efficient operation of network, but also is important to promote the healthy development of the national electric vehicle industries. 


\section{Review of Research at Home and Abroad}

Electric cars in China started relatively later than that in the developed countries, but the development is fast. To meet the need of the electric car development infrastructure's development, the provinces and energy companies are promote the building of charging infrastructure. In April 2010, the national standard of "Energy Consumption Experiment Method of Light Hybrid Electric Vehicle", "the General Requirements for Electric Vehicle Charging Station", "the Communication Protocol between the Electric Vehicle Battery Management System and the Non-Car Charger and the Conduction Charging Interface for Electric cars" were published. At the same time, supporting policies were carried out for new energy vehicles such as electric cars by state. At present, the electric car charging infrastructure has entered a stage of rapid large-scale implementation. And the influence of some running charging infrastructure for voltage, imbalance, loss, sag, voltage flicker, harmonic and so on, also appear constantly in power grid.

Currently, there is no mature mode and the comprehensive system research about forecast size of electric vehicles, randomness of user charging behavior, and the impact of access to grid and the corresponding charging control strategy. The research of impact on access to the grid that electric vehicle charging infrastructure includes the following contents: to assess whether the existing grid capacity can meet the demand of the growing electric vehicle load; to research the value of ancillary services that electric vehicle provides in its access to the grid including the frequency modulation, spinning reserve and so on; to research the increasing impact that the electric car has on medium and low voltage power grid which involved load, voltage, loss, unbalanced three-phase, harmonic and so on, but now the research results is less; and to carry out the relevant countermeasures on the influence above.

Currently, mathematical prediction methods are mainly to calculate the load forecasting, and its accuracy is affected by many factors. Some methods are commonly used, such as: gray model, regression analysis, exponential smoothing, time series, Kalman filtering and more advanced artificial intelligence methods such as artificial neural networks, expert systems, fuzzy prediction method. Given that, Monte Carlo method (also known as statistical simulation method) which is with relatively high precision and can simulate random factors is a class of mathematical numerical methods that are guided by statistical mathematical theory for solving the problems of computer simulation. Its principle is using the random distribution theory (or pseudo-random number) to solve a lot of random problems. The main advantage of Monte Carlo simulation is the ability to take full advantage of computer operation to perform mathematical problems. Currently, in accordance with national policy, some researchers study the charging-discharging mode and time of different types of electric vehicles, and take the Monte Carlo simulation methods to calculate electric vehicle loads, which reflect certain superiority. As the electric vehicles have not been large-scale accessed, the size and characteristics of the charging load are usually analyzed by simulation, while the construction of the electric vehicle charging load involves a variety of factors ,such as, characteristics of power battery charging, the behavior of electric vehicle users, charging methods.

\section{The Regional Total Load of Electric Vehicles and Prediction Curve of Charging Load}

The main types of electric vehicles in China are bus, taxi, public service vehicle and private car. According to "Electric Vehicle Conductive Interface" which is passed in April 2010, the charging mode is dived into slow charge, regular charge and fast charge. The electric vehicle' s development speed, scale and charging mode are affected by technological development speed, policies, market orientation, users' interest, environment and other factors. Considering factors which influence human's choices, a method based on intelligent ant colony algorithm is proposed. It can simulate Chinese different kinds of electric vehicle's ownership, development speed and scale in short term, medium and long term. So it can predict the total power load of electric vehicle in different periods and regions. 
The load curve of electric vehicle in prediction religion can be calculated by Monte Carlo. Then the power load curve of each electric vehicle will be accumulated to a total curve. The difficulties of charging load calculation is to analyze electric vehicle charging start time and the randomness.

The various charging types of electric vehicle can be classified according to the charging requirements. First, it is assumed that the power grid do not control the charging behavior, the electric vehicle will start charging when it connect to the grid. Using the Monte Carlo method to get the starting load state and charging time to calculate the electric vehicle's charging load. Then the characteristics of electric vehicle power load in different ownership can be predicted and analyzed.

Load forecasting model are varied to apply to different data structures. Therefore, choosing the correct load forecasting model is a crucial step. By selecting the appropriate technology, a predictive mathematical model for load forecasting is built. According to predicted value by prediction calculating or preliminary predictive value through other methods, with reference to the emerged possibilities and new trends, comprehensive analysis, comparison, judgment, reasoning, evaluation, finally, the preliminary forecast results are adjusted and amended. The reason is that the variable laws from the past to the present cannot be deemed to the variable laws in the future. So the new factors affecting the prediction target were analyzed to determine the predictive value with the appropriate amendments of prediction model.

Firstly, based on the analysis of the related factors of electric vehicles' development speed and human behavior, combined with characteristics of the power load forecasting and combination forecasting, this paper use one dimensional regression model, multiple linear regression model, gray prediction method to establish single prediction model of regional electricity charging load, Additionally, the accuracy of the individual models were compared. Secondly, the combination forecasting method is used to establish combination forecasting model of regional electric vehicle charging load. Using optimal weighting method to construct the objective function, intelligent ant colony algorithm to predict right weight of single prediction model in combination forecasting model, then, a single prediction model is re-screened based on the weight. Finally, the combination forecasting model are used to forecast and analysis the electricity load of regional electric vehicles in the short, medium and long term.

Firstly, according to the actual situation, electric vehicles will be classified in various types, then classifying charging types of electric vehicles and charging equipment structure, and building various charging models simulation of electric vehicles. Based on the total power load of electric vehicles in different periods, using Monte Carlo simulation method extract data samples, then effectively forecasting electric vehicle charging load curve in the region. The methods and procedures are shown in Fig. 1:

\section{Conclusion}

Using the above methods to predict charging load of the electric cars in a certain area, it is can be known that the period from 2016 to 2017 is initial stage of electric cars ,also can be called petit rate of growth stage, in which the growth of charging load is small and relatively fixed in charging time. 2018 to 2020, the electric vehicles charging load will increase rapidly and there will be the charging peak load. 2021-2030, with the popularity of private electric cars, electric vehicles charging load is increasing rapidly.

In the future, the electric cars will bring new load growth to power grid. Because of the characteristic of the car, the charging load has an obvious peak later and the reason is that private cars concentrated charging on weekdays and holiday night. Because the time of private car going home in holidays than working days more dispersed, the charging peak load declined in holiday. Electric vehicle charging load has obvious peak and valley difference, which peak load period and the whole network load peak load period are basically same. If the charging load of the electric vehicle will be controlled and be achieved effective peak shaving, then we can reduce the power supply and power grid investment and reduce the operation cost of power grid.

The electric vehicle charging load is influenced by many factors, and it is difficult to establish a mathematical model between the load and the factors. In this paper, the forecast method of electric 
vehicle charging load is explored. The forecast results have certain reference value for the study of the growth of China's electric vehicle charging load in the future.

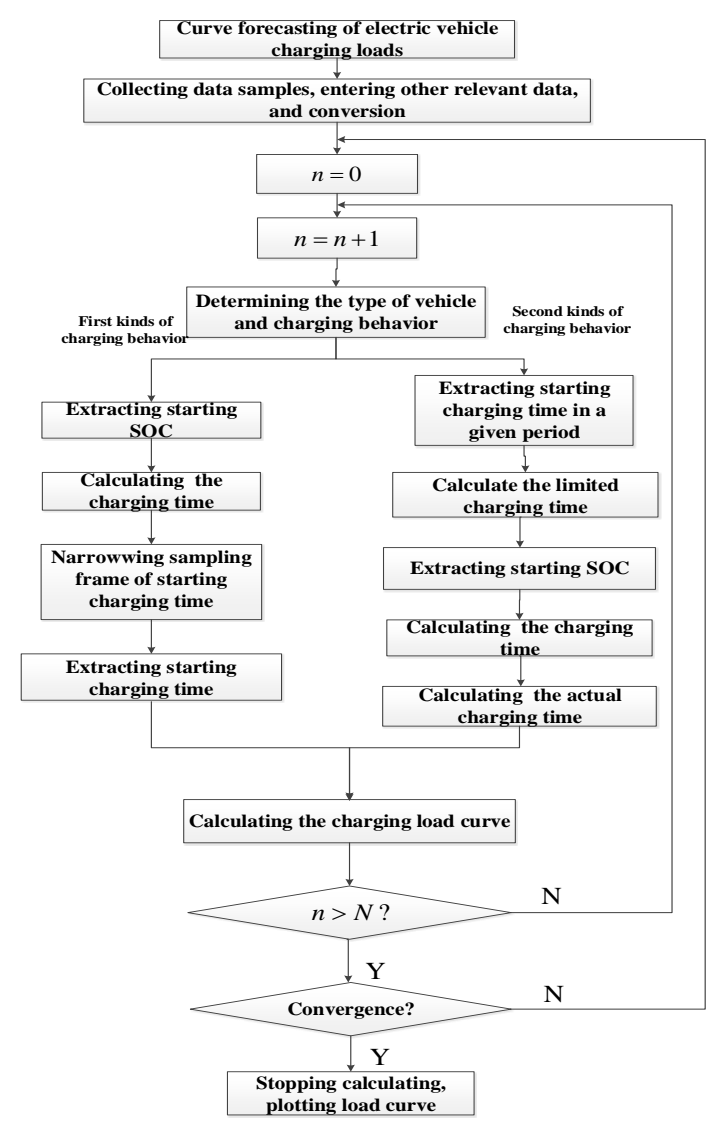

Figure 1. The methods and procedures

\section{References}

[1] Liu Wenxia, Pile Access Control Mode and Strategy for Substation Area [J].Automation of Electric Power Systems, 2013, 37(16): 66-72.

[2] YanXueming, YuanJinsha, Amodified particle swarm optimizer with dynamic adaptation [J]. Applied Mathematics and Computation, 2007, 189(2): 1205-1213.

[3] Yu Dayang, Song Shuguang, Synergisticdispatch of PEVs charging and wind power in chinese regional power grids [J].Automation of Electric Power Systems, 2011, 35(14): 24-29.

[4] Wu D, Aliprantis, Load scheduling and dispatch for aggregators of plug-in electric vehicles [J]. IEEE Trans on Smart Grid, 2011, 3(1): 368-376.

[5] Tian Liting, SHI Shuangtong, A statistical model for charging power demand of electric vehicles [J]. Power System Technology, 2010, 34(11): 126-130.

[6] Owen Worley, Diego KIabjall. Optimization of battery charging and purchasing at electric vehicle battery swap-Stations [C], vehicle Power and Propulsion Conference (vppc), 2011 IEEE, 20M: $1-4$.

[7] Wang Zhenpo, Liu Peng, Xin Tao. Optimizing the quantity of Off-broad charger for whole vehicle charging station $[\mathrm{C}], 2010$ International Conference on Optoelectronics and Image Processing (ICOIP), 2010:93-96.

[8] Thoralf Winkler, Przemyslaw Komarnieki, Gerhard Mud-ler. Electric vehicle charging stations in Magdeburg [c],Vehicle Power and Propulsion Conference(VPPC), 2009, IEEE, $200960-65$. 
[9] Deng Benzai, Wang Zhiqiang. Research on electric-vehicle charging station technologies based on smart grid [C], Asia-Pacific Power and Energy Engineering Conference (APPEEC), 2011: $1-4$.

[10]Gong Jing. Using wavelet packet decomposing coefficient to achieve distribution network single-phase ground fault line selection [J]. Power System Protection and Control, 2009, 24: 94-99. 